\title{
The chemical evolution of Barium and Europium in the Milky Way
}

\author{
Gabriele Cescutti ${ }^{1}$, Patrick François ${ }^{2}$, \\ and Francesca Matteucci ${ }^{1}$ \\ ${ }^{1}$ Dipartimento di Astronomia, Universitá di Trieste, via G.B. Tiepolo 11, I-34131 \\ email: cescutti@ts.astro.it \\ ${ }^{2}$ Observatoire de Paris/Meudon, GEPI, 61 Avenue de l'Observatoire, 75014 Paris, France
}

\begin{abstract}
We compute the evolution of the abundances of barium and europium in the Milky Way with a chemical evolution model which already reproduces the majority of observational constraints, and we compared our results with the observed abundances from the recent UVES Large Program "First Stars" (Cayrel et al. 2004, François et al. 2005).

We confirm that barium is a neutron capture element mainly produced in the low mass AGB stars, during the thermal-pulsing phase, by the ${ }^{13} \mathrm{C}$ neutron source, in a slow neutron capture process. However, in order to reproduce the $[\mathrm{Ba} / \mathrm{Fe}]$ vs. $[\mathrm{Fe} / \mathrm{H}]$ as well as the Ba solar abundance, Ba should be also produced as a r-process element by massive stars in the range $10-30 M_{\odot}$. On the other hand, europium should be only a r-process element produced in the same range of masses $\left(10-30 M_{\odot}\right)$, at variance with previous suggestions indicating a smaller mass range for the Eu producers. As it is well known, there is a large spread in the $[\mathrm{Ba} / \mathrm{Fe}]$ and $[\mathrm{Eu} / \mathrm{Fe}]$ ratios at low metallicities, although smaller in the newest data. With our model we estimate ranges in the r-process yields from massive stars for both elements which better reproduce the trend of the data. We discuss several possibilities to explain the observed spread. We suggest that a peculiar behaviour of the neutron capture elements could be the responsible for the spread instead of invoking a strongly inhomogeneous early Galactic halo. We finally underline that the production ratio of $[\mathrm{Ba} / \mathrm{Eu}]$ may be almost constant in the massive stars.
\end{abstract}

Keywords. Nuclear reactions, nucleosynthesis, abundances, stars: abundances, Galaxy: abundances

\section{Introduction}

The neutron capture is the main mechanism to form elements heavier than iron. The other mechanism, the p-process, is required for the proton rich isotopes, whose abundances in the solar system is less than $1 \%$. Two major neutron capture mechanisms are generally invoked: the slow process (s-process) and the rapid process (r-process), where the slow and rapid are defined relatively to the timescale of $\beta$-decay. The s-process requires a relatively low neutron density and moves along the valley of $\beta$ stability. The site of production of the s-elements is not unique. In fact, the main component, accounting for the s-process in the atomic mass number range $90<A<208$, was shown to occur in the low-mass asymptotic giant branch (AGB) stars during recurrent thermal pulses (Gallino et al. 1998; Busso et al. 1999). On the other hand, the weak s-component is responsible for the s-process nuclides up to $A \simeq 90$ and it is recognized as the result of neutron capture in advanced evolution in massive stars (see Raiteri et al. 1993). Finally, the strong-s component was introduced by Clayton \& Rassbach(1967) in order to reproduce more than $50 \%$ of the solar ${ }^{208} \mathrm{~Pb}$.

The r-process takes place in extremely neutron-rich environments in which the mean time between two successive neutron captures is very short, compared with the time necessary for the $\beta$-decay. Several scenarios have been proposed for the origin of r-process elements: neutrino winds in core-collapse supernovae (Woosley et al. 1994), the collapse of ONeMg cores resulting from stars with initial masses in the range $8-10 M_{\odot}$ (Wanajo et al. 2003) and neutron star 
mergers (Freiburghaus et al. 1999). However, no clear consensus has been achieved and r-process nucleosynthesis remains still uncertain.

In order to shed light on the nature (s- and/or r- processes) of heavy elements such as Ba and $\mathrm{Eu}$ one should examine the abundances of these elements in Galactic stars of all metallicities. These abundances can, in fact, give us clues to interpret their nucleosynthetic origin.

One striking aspect of the data relative to both $\mathrm{Ba}$ and $\mathrm{Eu}$ is the large spread observed in the $[\mathrm{Ba} / \mathrm{Fe}]$ and $[\mathrm{Eu} / \mathrm{Fe}]$ ratios in halo stars (e.g. Mc William et al. 1995; Ryan et al. 1996). We compare the predictions relative to the evolution of $\mathrm{Ba}$ and Eu with the newest data of François et al. (2005). A spread, although lower than in previous data, still exists for $[\mathrm{Ba} / \mathrm{Fe}]$ and $[\mathrm{Eu} / \mathrm{Fe}]$ at low metallicities. We attempt to give an explanation of this spread without invoking only the inhomogeneous halo mixing.

\section{Observational data}

We preferentially used the most recent available data based on high quality spectra collected with efficient spectrographs and 8-10 m class telescopes. In particular, for the extremely metal poor stars $([\mathrm{Fe} / \mathrm{H}]$ between -4 and -3$)$, we adopted the recent results from UVES Large Program "First Star" (Cayrel et al. 2004, François et al. 2005). This sample is made of 31 extremely metal-poor halo stars selected in the HK survey (Beers et al. 1992, 1999). We can deduce from the kinematics of these stars that they were born at very different places in the Galactic halo. This overcomes the possibility of a selection bias. The analysis is made in a systematic and homogeneous way, from very high quality data, giving abundance ratios of unprecedented accuracy in this metallicity range. For the abundances in the remaining range of $[\mathrm{Fe} / \mathrm{H}]$, we took published high quality data in the literature from various sources: McWilliam et al. (1995), Ryan et al. (1996), Beers et al. (1999), Fulbright(2000), Mashonkina \& Gehren (2000, 2001), Koch \& Edvardsson(2002), Honda et al. (2004), Ishimaru et al. (2004). All of this data are relative to solar abundances of Grevesse \& Sauval (1998).

\section{Chemical evolution model for the Milky Way}

We model the formation of the Galaxy assuming two main infall episodes: the first forms the halo and the thick disk, the second the thin disk. The timescale for the formation of the halo-thick disk is $\sim 1 G y r$. The timescale for the thin disk is much longer $(\sim 7 G y r)$, implying that the infalling gas forming the thin disk comes mainly from the intergalactic medium and not only from the halo (Chiappini et al. 1997). A threshold in the star formation process (Martin $\&$ Kennicutt 2001) is also adopted. The model well reproduces an extended set of observational constraints both for the solar neighborhood and for the whole disc. One of the most important observational constraints is represented by the various relations between the abundances of metals (C,N,O, $\alpha$-elements, iron peak elements) as functions of the $[\mathrm{Fe} / \mathrm{H}]$ abundance.

\section{Nucleosynthesis Prescriptions}

We have adopted the yields of Busso et al. (2001) in the mass range 1.5-3M $\odot$ for the smain component. In this process, the dependence on the metallicity is very important. In fact, the s-process elements are made by accretion of neutrons on seed elements (in particular iron) already present in the star. Therefore, this Ba component behaves like a secondary element. The theoretical results by Busso et al. (2001) suggest negligible Europium production in the s-process and therefore we neglected this component in our work.

We have assumed for $\mathrm{Ba}$ and $\mathrm{Eu}$ a r-process component, produced in massive stars in the range $10-30 M_{\odot}$ (see table 1$)$. In particular, our choice is done with the purpose of best fitting the plots $[\mathrm{Ba} / \mathrm{Fe}]$ vs. $[\mathrm{Fe} / \mathrm{H}],[\mathrm{Eu} / \mathrm{Fe}]$ vs. $[\mathrm{Fe} / \mathrm{H}]$ and $[\mathrm{Ba} / \mathrm{Eu}]$ vs $[\mathrm{Fe} / \mathrm{H}]$ as well as the $\mathrm{Ba}$ and $\mathrm{Eu}$ solar abundance (taking into account the contribution of the low-intermediate mass star in case of the Ba). 
Table 1. The stellar yields for Barium and Europium in massive stars (r-process).

\begin{tabular}{|c|c|c|}
\hline$M_{\text {star }}$ & $X_{B a}^{\text {new }}$ & $X_{E u}^{\text {new }}$ \\
\hline 10. & $9.00 \cdot 10^{-7}$ & $4.50 \cdot 10^{-8}$ \\
15. & $3.00 \cdot 10^{-8}$ & $3.00 \cdot 10^{-9}$ \\
30. & $1.00 \cdot 10^{-9}$ & $5.00 \cdot 10^{-10}$ \\
\hline
\end{tabular}
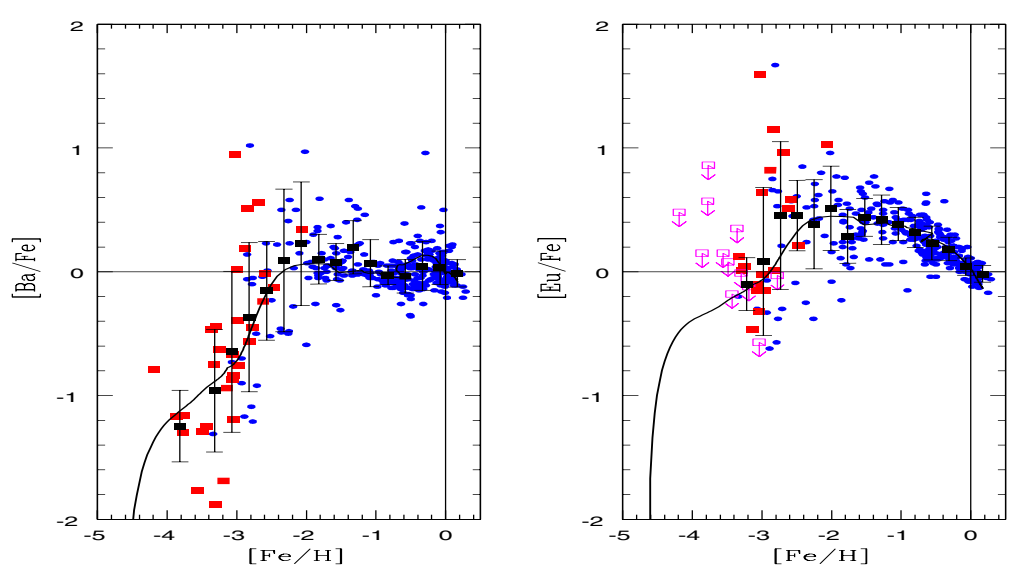

Figure 1. On the left side is plotted the $[\mathrm{Ba} / \mathrm{Fe}]$ versus $[\mathrm{Fe} / \mathrm{H}]$ and on the right the $[\mathrm{Eu} / \mathrm{Fe}]$ versus $[\mathrm{Fe} / \mathrm{H}]$ for the data by François et al. (2005) (in filled squares the data, in open squares the upper limits) and for other observational data (see Sect. 2, the filled circles). The squares with error bars are the mean values of the data bins and the error bars are the standard deviation. We show in solid line the results of our model.

Table 2. Solar abundances of $\mathrm{Ba}$ and $\mathrm{Eu}$, as predicted by our models, compared with the observed ones from Grevesse \& Sauval (1998).

\begin{tabular}{|c|c|c|c|c|}
\hline$\left(X_{B a}\right)_{p r}$ & $\mid \% B a_{s} / B a$ & $X_{B a \odot}$ & $\left(X_{E u}\right)_{p r}$ & $X_{E u \odot}$ \\
\hline $1.55 \cdot 10^{-8}$ & $54 \%$ & $1.62 \cdot 10^{-8}$ & $4.06 \cdot 10^{-10}$ & $3.84 \cdot 10^{-10}$ \\
\hline
\end{tabular}

For the nucleosynthesis prescriptions of the Fe, we adopted those suggested in François et al. (2004).

\section{Results}

\subsection{Trends}

In this Section we investigate how the different models fit the the trends of the abundances ratios for $[\mathrm{Ba} / \mathrm{Fe}],[\mathrm{Eu} / \mathrm{Fe}]$ and $[\mathrm{Ba} / \mathrm{Eu}]$ versus $[\mathrm{Fe} / \mathrm{H}]$ and even for $[\mathrm{Ba} / \mathrm{Eu}]$ versus $[\mathrm{Ba} / \mathrm{H}]$. To better understand the trends of the data we have decided to divide in several bins the $[\mathrm{Fe} / \mathrm{H}]$ axis and the $[\mathrm{Ba} / \mathrm{H}]$ axis and compute the mean and the standard deviation from the mean of the ratios $[\mathrm{Ba} / \mathrm{Fe}],[\mathrm{Eu} / \mathrm{Fe}]$ and $[\mathrm{Ba} / \mathrm{Eu}]$ for all the data inside each bin.

As one can see in Figure 1, where we have plotted the predictions of our model for [Ba/Fe] versus $[\mathrm{Fe} / \mathrm{H}]$, the model fits quite well the trend of the data. In these models the upper mass limit for the production of the r-processed $\mathrm{Ba}$ is $30 M_{\odot}$.

On the right side of the Figure 1, we show the results of our model in this case for [Eu/Fe] versus $[\mathrm{Fe} / \mathrm{H}]$. The trend of the data is also well reproduced by the model from low metallicity to the solar metallicity. 

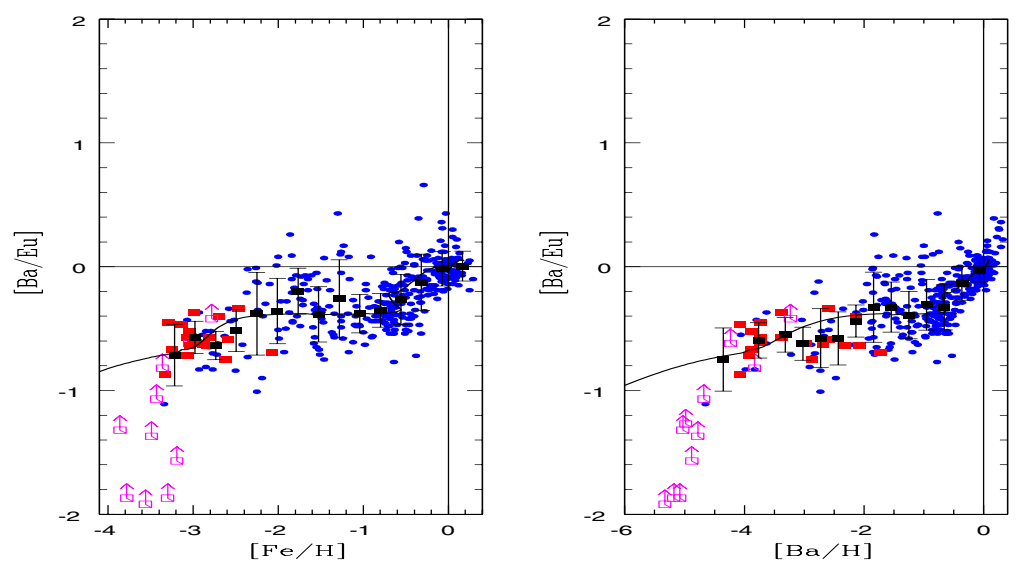

Figure 2. On the left side we show the ratio $[\mathrm{Ba} / \mathrm{Eu}]$ versus $[\mathrm{Fe} / \mathrm{H}]$, on the right side the ratio $[\mathrm{Ba} / \mathrm{Eu}]$ versus $[\mathrm{Ba} / \mathrm{H}]$ for the data by François et al. (2005) (in filled squares the data, in open squares the lower limits) and for other observational data (see Sect. 2, the filled circles). The squares with error bars are the mean values of the data bins and as error bars we consider the standard deviation. The results of the model are represented in solid line.

In table 2 we show the predicted solar abundances of $\mathrm{Eu}$ and $\mathrm{Ba}$ for our model compared to the solar abundances by Grevesse \& Sauval (1998). We have put in the table also the predicted s-process fraction in the Barium solar abundance. The results of our model are in good agreement with the solar abundances but note that we predict a slightly different s-process fraction (nearly $60 \%$ instead of $80 \%$ ) compared with the s-process fraction obtained by previous authors (Travaglio et al. 1999, Arlandini et al. 1999, Raiteri et al. 1992 and Käppeler et al. 1989). This different result is due to the different adopted chemical evolution model.

If we look at Fig. 2 where we have plotted the abundances of $[\mathrm{Ba} / \mathrm{Eu}]$ versus $[\mathrm{Fe} / \mathrm{H}]$ and $[\mathrm{Ba} / \mathrm{Eu}]$ versus $[\mathrm{Ba} / \mathrm{H}]$, we note two important features. The first feature is that it is evident from the data that there is a plateau in the $[\mathrm{Ba} / \mathrm{Eu}]$ ratio before the production of s-process $\mathrm{Ba}$ by the low intermediate mass stars starts to be non negligible at $[\mathrm{Fe} / \mathrm{H}] \sim-1$ and $[\mathrm{Ba} / \mathrm{H}] \sim$ -0.8 . Moreover, the timescale of rise of the $[\mathrm{Ba} / \mathrm{Eu}]$ value, due to the production of $\mathrm{Ba}$ by low intermediate mass stars, is very well reproduced by our model. The second feature is that the spread in the ratio of $[\mathrm{Ba} / \mathrm{Eu}]$ both versus $[\mathrm{Fe} / \mathrm{H}]$ and $[\mathrm{Ba} / \mathrm{H}]$ is significantly lower than the spread in $[\mathrm{Ba} / \mathrm{Fe}]$, in particular when using as evolutionary tracer the $[\mathrm{Ba} / \mathrm{H}]$. Because of this we suggest that the mechanism which produces the observational spread does not affect the ratio of these two elements. In our picture, the explanation of the significantly smaller spread in the ratio of $[\mathrm{Ba} / \mathrm{Eu}]$ is that the site of production of these two elements is the same, the neutronized shell close to the mass cut in a SNII (see Woosley et al. 1994). The difference could be the amount of the neutronized material that each massive star expells during the SNII explosion. In fact, the mass cut and also the ejected neutronized material, are still uncertain quantities and usually they are considered as parameters in the nucleosynthesis codes for massive stars (see Rauscher et al. 2002, Woosley \& Weaver 1995, Woosley et al. 1994).

\subsection{Upper and lower limit to the r-process production and the possible explanations for the observed spread}

The purpose of this Section is to give upper and lower limits to the yields in order to contain the observed spread at low metallicities for Ba and Eu. Our aim is to understand the possible causes for this spread and give useful constraints to stellar nucleosynthesis. To do this we have increased the yields of the model for both elements ( $\mathrm{Ba}$ and $\mathrm{Eu}$ ), leaving untouched the s-process yields and changing only the yields of the r-process. We did that in order to obtain a prediction of our model which could be considered as an upper limit to the observational data. Then, to 
Table 3. The stellar yields for model Max and Min for Barium and Europium in massive stars (r-process).

\begin{tabular}{|c|c|c|c|c|}
\hline & \multicolumn{2}{|c|}{ Model Max $\mid$ Model Min } & \multicolumn{2}{|c|}{ Model Max | Model Min } \\
\hline$M_{\text {star }}$ & $X_{B a}^{\text {new }}$ & $X_{B a}^{\text {new }}$ & $X_{\text {Eu }}^{\text {new }}$ & $X_{E u}^{\text {new }}$ \\
\hline 12. & $1.35 \cdot 10^{-7}$ & $4.50 \cdot 10^{-8}$ & $4.50 \cdot 10^{-8}$ & $2.25 \cdot 10^{-8}$ \\
\hline$<15$. & $4.50 \cdot 10^{-8}$ & $1.50 \cdot 10^{-8}$ & $3.00 \cdot 10^{-9}$ & $1.50 \cdot 10^{-9}$ \\
\hline$\geqslant 15$ & $1.00 \cdot 10^{-7}$ & $5.00 \cdot 10^{-10}$ & $3.00 \cdot 10^{-8}$ & $1.50 \cdot 10^{-10}$ \\
\hline 30. & $1.00 \cdot 10^{-7}$ & $5.00 \cdot 10^{-10}$ & $5.00 \cdot 10^{-8}$ & $2.50 \cdot 10^{-10}$ \\
\hline
\end{tabular}
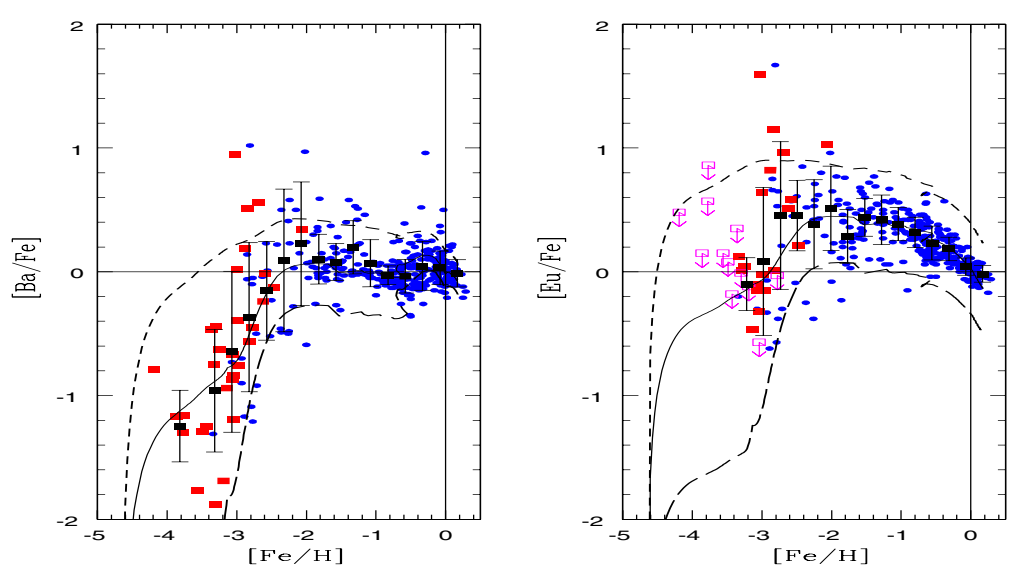

Figure 3. We show the ratio $[\mathrm{Ba} / \mathrm{Fe}]$ versus $[\mathrm{Fe} / \mathrm{H}]$ on the left side and the ratio $[\mathrm{Eu} / \mathrm{Fe}]$ versus $[\mathrm{Fe} / \mathrm{H}]$ on the right side for the data as in Fig. 1 . The solid line is the prediction of the best model, the short dashed line is the prediction of model Max and the long dashed line is the prediction of model min.

obtain a lower limit, the yields have been decreased. The two new resulting models have been called Max and Min and their characteristics are summarized in the table 3.

In Fig. 3 we plot the $[\mathrm{Ba} / \mathrm{Fe}]$ vs $[\mathrm{Fe} / \mathrm{H}]$ and $[\mathrm{Eu} / \mathrm{Fe}]$ vs $[\mathrm{Fe} / \mathrm{H}]$ relations for the new models Max and Min compared to the observational data. The two curves can well contain the observed spread for both Ba and Eu. In fact, with the chosen prescriptions only less than the $10 \%$ of the data does not fall inside the area contained by two models. We want to underline the fact that to obtain the upper limit we have to change by a factor of 10 the value of the yields in the more massive stars $\left(15-30 M_{\odot}\right)$ both for Barium and Europium; instead we have increased the yields in the range $12<M_{\odot}<15$ only of a factor of 1.5 for $\mathrm{Ba}$ and we have not changed them for Eu. We have operated in a similar way with the lower limit model; in fact, in the range $12<M_{\odot}<15$ the factor we used is 0.5 both for Eu and Ba. In the range $15-30 M_{\odot}$ the factor is much lower: 0.05. We can deduce from these upper and lower limit models that the spread could be due to a different production of heavy elements among massive stars $\left(>15 M_{\odot}\right)$. This type of stars could produce different amounts of these elements independently of the mass. As we have introduced in the previous subsect. (5.1), it is possible to link this fact with the problems of mass cut and the fall back during the explosion of a SNII. If these elements are produced in a shell close to the iron core of the star, differences in the explosion behaviour can give rise to a different quantity of r-process elements expelled by the star. This explanation suggests that the ratio of $\mathrm{Ba} / \mathrm{Eu}$ produced by massive stars could be nearly the same and only the total amount of r-process elements could change from star to star even of the same mass. 


\section{Conclusions}

Comparing the results of our chemical evolution model with the new observational data we can conclude that:

- Barium needs two components: a s-process main component originating in low mass stars plus a r-component originating in stars in the range $10-30 M_{\odot}$.

- Europium is mainly produced by a r-process and stars in the mass range $10-30 M_{\odot}$ should be considered as the only progenitors of this element.

We have discussed the spread observed in $[\mathrm{Ba} / \mathrm{Fe}]$ and $[\mathrm{Eu} / \mathrm{Fe}]$ at low metallicities and proposed that is probably due to a differential production of heavy elements in massive stars. In order to conclude that we have artificially varied the yields of both elements either as functions of mass or metallicity. The nearly constant value of the ratio $[\mathrm{Ba} / \mathrm{Eu}]$ produced in massive stars by the r-process, can be used to estimate the fraction of Barium in the solar abundance produced by the slow process. We have obtained in this way a fraction that is different from the previous estimates: $60 \%$ instead of $80 \%$. The yields that we derived, in particular the limits that we are able to give and also the fact that the ratio of the r- process production of $\mathrm{Eu}$ and $\mathrm{Ba}$ must be nearly constant, could be very useful to studies involving nucleosynthesis in stellar models and even to nuclear physics studies.

\section{References}

Arlandini, C., Käppeler, F., Wisshak, K. et al., 1999, ApJ, 525, 886

Beers, T.C., Rossi, S., Norris, J.E., Ryan, S.G., \& Shefler, T., 1999, AJ 117, 981

Busso, M., Gallino, R., Lambert, D.L., Travaglio, C., \& Smith, V.V., 2001, ApJ, 557, 802

Busso, M., Gallino, R., \& Wasserburg, G.J., 1999, ARA\&A,37, 239

Cayrel, R., Depagne, E., Spite, M. et al., 2004, A\&A A, 416, 1117

Chiappini, C., Matteucci, M.F., \& Gratton, R.G., 1997, ApJ, 477, 765

Chiappini, C., Romano, D., \& Matteucci, M.F., 2003, MNRAS, 339, 63

Clayton, D.D. \& Rassbach, M.E., 1967, ApJ, 168, 69

François, P., Depagne, E., Hill, V. et al., 2005, in preparation

François, P., Matteucci, F., Cayrel, R. et al., 2004, A\& A, 421, 613

Freiburghaus, C., Rosswog, S., \& Thielemann, F.-K., 1999, ApJ, 525L, 121

Fulbright, J.P., 2000, AJ, 120, 1841

Gallino, R., Arlandini, C., Busso, M. et al., 1998, ApJ, 497, 388

Grevesse, N. \& Sauval, A.J., 1998, Space Sci. Rev., 85, 161

Honda, S., Aoki, W., Kajino, T. et al., 2004, ApJ, 607, 474

Ishimaru, Y., Wanajo, S., Aoki, W., \& Ryan, S.G., 2004, ApJ, 600L, 47

Käppeler, F., Beer, H., \& Wisshak, K., 1989, Rep.Prog.Phys., 52, 945

Koch, A. \& Edvardsson, B., 2002, A\& A, 381, 500

Martin, C.L. \& Kennicutt, R.C.Jr., 2001, ApJ, 555, 301

Matteucci, M.F. \& François, P., 1989, MNRAS, 239, 885

Matteucci, M.F. \& Greggio, L., 1986, A\&A A, 154, 279

Mashonkina, L. \& Gehren, T., 2000, A\&A, 364, 249

Mashonkina, L. \& Gehren, T., 2001, A\& A , 376, 232

McWilliam, A., Preston, G.W., Sneden, C. \& Searle, L., 1995, AJ, 109, 2757

Raiteri, C.M., Gallino, R., \& Busso, M., 1992, ApJ, 387, 263

Raiteri, C.M., Gallino, R., \& Busso, M., 1993, ApJ, 419, 207

Rauscher, T., Heger, A., Hoffmann, R.D., \& Woosley, S.E., 2002, ApJ, 576, 323

Ryan, S., Norris, J.E. \& Beers T.C., 1996, ApJ, 471, 254

Travaglio, C., Galli, D., Gallino, R. et al., 1999, ApJ, 521, 691

Travaglio, C., Galli, D. \& Burkert, A., 2001, ApJ, 547, 217

Wanajo, S., Tamamura, M., Itoh, N. et al., 2003, ApJ, 593, 968

Woosley, S.E. \& Hoffmann, R.D., 1992, ApJ, 395, 202

Woosley, S.E. \& Weaver, T.A., 1995, ApJ, 101, 181

Woosley, S.E., Wilson, J.R., Mathews, G.J., Hoffman, R.D., \& Meyer, B.S., 1994, ApJ, 433, 229 